\title{
Role of the pair potential for the saturation of generalized Pauli constraints
}

\author{
Örs Legeza ${ }^{1}$ and Christian Schilling ${ }^{2}, *$ \\ ${ }^{1}$ Wigner Research Centre for Physics, Hungarian Academy of Sciences, \\ Konkoly-Thege Miklós út 29-33, 1121 Budapest, Hungary \\ ${ }^{2}$ Clarendon Laboratory, University of Oxford, Parks Road, Oxford OX1 3PU, United Kingdom
}

(Dated: June 2, 2021)

\begin{abstract}
The dependence of the (quasi-)saturation of the generalized Pauli constraints on the pair potential is studied for ground states of few-fermion systems. For this, we consider spinless fermions in one dimension which are harmonically confined and interact by pair potentials of the form $\left|x_{i}-x_{j}\right|^{s}$ with $-1 \leq s \leq 5$. We use the Density Matrix Renormalization Group approach and large orbital basis to achieve the convergence on more than ten digits of both the variational energy and the natural occupation numbers. Our results confirm that the conflict between energy minimization and fermionic exchange symmetry results in a universal and non-trivial quasi-saturation of the generalized Pauli constraints (quasipinning), implying tremendous structural simplifications of the fermionic ground state for all $s$. Those numerically exact results are complemented by an analytical study based on a self-consistent perturbation theory which we develop for this purpose. The respective results for the weak coupling regime eventually elucidate the singular behaviour found for the specific values $s=2,4, \ldots$, resulting in an extremely strong quasipinning.
\end{abstract}

PACS numbers: 03.65.-w, 05.30.Fk, 31.15.ac

\section{INTRODUCTION}

In recent years, quantum information theoretical concepts began to play a more important role in the description and understanding of quantum many-body systems. One of the prime examples reflecting the progress at that exciting interface and its fruitful future prospects is given by Klyachko's breakthrough result a few years ago: It has been shown that Pauli's original exclusion principle [1] - despite its long-term success on all physical length scales - is incomplete. Indeed, as already suggested by first studies [2, 3, the fermionic exchange symmetry has been found [4 6] to imply more restrictive constraints on the one-particle picture, independent of the underlying Hamiltonian, rendering Pauli's original principle obsolete. To be more precise, these so-called generalized Pauli constraints (GPC) take the form of linear conditions,

$$
D_{j}(\vec{\lambda}) \equiv \kappa_{j}^{(0)}+\sum_{k=1}^{d} \kappa_{j}^{(k)} \lambda_{k} \geq 0,
$$

on the decreasingly-ordered natural occupation numbers $\vec{\lambda} \equiv\left(\lambda_{k}\right)_{k=1}^{d}$, the eigenvalues of the one-particle reduced density operator $\rho_{1} \equiv N \operatorname{Tr}_{N-1}\left[\left|\Psi_{N}\right\rangle\left\langle\Psi_{N}\right|\right]$. Here, $\left|\Psi_{N}\right\rangle \in \wedge^{N}\left[\mathcal{H}_{1}^{(d)}\right]$ is the $N$-fermion quantum state, where the one-particle Hilbert space $\mathcal{H}_{1}^{(d)}$ has dimension $d$ and $j=1, \ldots, \nu^{(N, d)}, \kappa_{j}^{(k)} \in \mathbb{Z}$. For each setting $(N, d)$, the finite set of GPCs defines a convex polytope $\mathcal{P} \subset \mathbb{R}^{d}$, a subset of the Pauli simplex $1 \geq \lambda_{1} \geq \ldots \geq \lambda_{d} \geq 0$. Moreover, the values $D_{j}(\vec{\lambda})$ coincide up to a prefactor with the $l_{1}$-distances of $\vec{\lambda}$ to the respective polytope facets $F_{j}$ corresponding to $D_{j} \equiv 0[7$.

\footnotetext{
* christian.schilling@physics.ox.ac.uk
}

In complete analogy to Pauli's original exclusion principle, the potential physical relevance of the GPCs would primarily be based on their saturation in concrete systems. Such pinning would then reduce the complexity of the system's quantum state and would define " $a$ new physical entity with its own dynamics and kinematics" [8] (see also [9, 10]). Moreover, the geometrical structure underlying the GPCs suggests a natural hierarchy of extensions of the Hartree-Fock ansatz allowing one to systematically capture static correlations in few-body quantum systems [10].

In an analytical study [11] of three harmonically interacting spinless fermions in a one-dimensional harmonic trap it has been shown that the GPCs are saturated up to a very small correction of the order eight in the dimensionless coupling, $D \propto \kappa^{8}$. A succeeding extended study of such harmonic models [7, 12, 16 has confirmed, by varying the particle number, spatial dimension, degree of spin-polarization and coupling strength that this striking quasipinning-effect has a physical origin. It namely emerges from the conflict between energy minimization and fermionic exchange symmetry 14. The intensive ongoing debate discussing the physical relevance of the GPCs in more realistic systems, as, e.g., atoms and molecules (see [8, 9, 13, 17 33 and references therein) has been hampered, however, due to a couple of reasons: First, most of the numerical studies of realistic systems were based so far on very small active spaces of 3-5 orbitals and may therefore fail to accurately capture the true physical situation. Second, it has only been realized very recently that (quasi)pinning is in some cases trivial in the sense that it is a mere consequence of spatial symmetries 21, 25, 27) or may just follow from an (approximate) saturation of the Pauli constraints [15, 19].

In the present work, we revisit the quasipinning phenomenon by addressing all the concerns mentioned 
above. We namely use state-of-the-art numerical methods to calculate the natural occupation numbers to a high precision and use a concise quantitative measure to distinguish trivial from non-trivial (quasi)pinning. The main aim is thus to estimate the genuine relevance that GPCs have in few-fermion quantum systems. By considering one-dimensional harmonic trap systems of fully-polarized fermions, we ensure that possible (quasi)pinning is neither an artifact of orbital-symmetries nor of spin-symmetries. On the other hand, freezing degenerate angular and spin degrees of freedom and considering a steep external trap reduces the size of the underlying active space and thus allows us to eventually perform a fully conclusive analysis of the GPCs' relevance in those systems. This is quite different in atoms, where the Coulomb interaction between the electrons manifests itself in a wave function cusp which entails the presence of dynamic correlations. While those dynamic correlations typically do not change the qualitative physical behavior, their recovering with high precision requires, however, much larger active spaces for which the generalized Pauli constraints are not known yet. We therefore believe that our succeeding analysis will give us a rather good idea of the genuine relevance that GPCs have in few-fermion quantum systems.

The Hamiltonians at hand take the form

$$
\hat{H}=\sum_{j=1}^{N}\left(\frac{\hat{p}_{j}^{2}}{2 m}+\frac{1}{2} m \omega^{2} \hat{x}_{j}^{2}\right)+K \sum_{j<k}\left|\hat{x}_{j}-\hat{x}_{k}\right|^{s} .
$$

By using the natural length scale of the external harmonic trap, $l \equiv \sqrt{\hbar / m \omega^{2}}$, we introduce the dimensionless coupling parameter

$$
\kappa \equiv K / m \omega^{2} l^{2-s} .
$$

This is equivalent to just setting $m \equiv \omega \equiv \hbar \equiv 1$. Besides the general motivation above, there are further important reasons for choosing the family of Hamiltonians of the form (2) rather than the electronic Hamiltonians studied in quantum chemistry: The remarkable recent progress in quantum optics allows one to simulate with ultracold gases an increasing variety of physical systems and models which high flexibility and control. For instance, in contrast to the electrons in atoms and molecules, the interaction between the ultracold fermionic atoms can be tuned at the Feshbach resonance [34 36]. Furthermore, by departing from the dilute gas regime, the effective pair interaction between the fermionic atoms is typically described by a Lennard-Jones-type potential, and thus differs not that much anymore from (2). The future prospects of quantum simulation in general and the proposed experimental realization of the (quasi)pinningeffect in systems of ultracold fermionic atoms in particular (see, e.g., the expected "transparency effect" [25]) provide further compelling reasons for studying the GPCs in harmonic trap systems rather than in weaklycorrelated few-electron atoms.
In the following section, we develop a self-consistent perturbation theoretical approach to determine the leading order behavior of the NONs in the regime of weak coupling. In Section III we then explain how to obtain the numerically exact ground state of (2) for finite coupling by using the Density Matrix Renormalization Group (DMRG) approach 37. Eventually, we use those results to discuss in Section IV the relevance of the GPCs for different Hamiltonians (2) by considering about 100 different $s$-values.

\section{ANALYTICAL APPROACH FOR WEAK COUPLING}

In this section we elaborate on a perturbation theoretical approach to determine the leading order behaviour of the minimal distance $D(\vec{\lambda}(\kappa))$ of $\vec{\lambda}$ to the polytope boundary for the ground state $|\Psi(\kappa)\rangle$ of a general $N$ particle Hamiltonian,

$$
\hat{H}(\kappa)=\hat{H}^{(0)}+\kappa \hat{V},
$$

in the regime of weak coupling. Here, $\hat{H}^{(0)}$ denotes the one-particle Hamiltonian, $\hat{V}$ the pair interaction and the Hamiltonian acts on the $N$-fermion Hilbert space $\wedge^{N}\left[\mathcal{H}_{1}^{(d)}\right]$, where we assume the one-particle Hilbert space to be finite, $d$-dimensional. We assume that the ground state is non-degenerate and that the respective $D(\vec{\lambda}(\kappa))$ is analytical in $\kappa$, at least in a neighbourhood of $\kappa=0$. Since $D(\vec{\lambda}(0))=0$, following from the fact that $\vec{\lambda}(0)=$ $(1, \ldots, 1,0, \ldots, 0)$ is a vertex of the polytope ("HartreeFock point"), and $D(\vec{\lambda}(\kappa)) \geq 0$ for all $\kappa$, the linear order of $D(\vec{\lambda}(\kappa))$ must vanish and therefore the leading order correction is quadratic. Determining this second order term by exploiting conventual perturbation theory is a rather lengthy exercise. In a first step, one would need to determine $|\Psi(\kappa)\rangle$ up to order $\kappa^{2}$. Then, one would need to determine the one-particle reduced density operator,

$$
\begin{aligned}
\rho_{1}(\kappa) & \equiv N \operatorname{Tr}_{N-1}[|\Psi(\kappa)\rangle\langle\Psi(\kappa)|] \\
& \equiv \sum_{j=1}^{d} \lambda_{j}(\kappa)\left|\varphi_{j}(\kappa)\right\rangle\left\langle\varphi_{j}(\kappa)\right|,
\end{aligned}
$$

of $|\Psi(\kappa)\rangle$ by tracing out $N-1$ fermions. Recall that the natural occupation numbers $\lambda_{j}(\kappa)$ shall be ordered nonincreasingly and the respective natural orbitals $\left|\varphi_{j}(\kappa)\right\rangle$ are uniquely defined as long as the natural occupation numbers are non-degenerate. Finally, one would need to perform again second order perturbation theory, this time on $\rho_{1}(\kappa)$, to determine the natural occupation numbers up to corrections of $\mathcal{O}\left(\kappa^{2}\right)$. This last step is particularly challenging since it involves degenerate unperturbed eigenvalues. Indeed, one has $\operatorname{spec}\left(\rho_{1}(0)\right)=$ $(1, \ldots, 1,0, \ldots, 0)$. In the following we therefore elaborate on a self-consistent perturbation theory, which may simplify the task quite a lot. 


\section{A. Self-consistent perturbation theory}

The crucial point of our perturbation theoretical approach is that it exploits a self-consistent expansion of $|\Psi(\kappa)\rangle$. We namely expand $|\Psi(\kappa)\rangle$ for each coupling $\kappa$ as a linear combination of the Slater determinants built from its own natural orbitals [38,

$$
|\Psi(\kappa)\rangle=\sum_{i} c_{i}(\kappa)|\boldsymbol{i}(\kappa)\rangle
$$

Here, we use the shorthand notation

$$
|\boldsymbol{i}(\kappa)\rangle \equiv\left|\varphi_{i_{1}}(\kappa), \ldots, \varphi_{i_{N}}(\kappa)\right\rangle
$$

for the respective $N$-particle Slater determinant constructed from the $N$ natural orbitals $\left|\varphi_{i_{1}}(\kappa)\right\rangle, \ldots,\left|\varphi_{i_{N}}(\kappa)\right\rangle$ and $\boldsymbol{i} \equiv\left(i_{1}, \ldots, i_{N}\right)$. Moreover, $|\Psi(\kappa)\rangle$ is normalized to unity, $\langle\Psi(\kappa) \mid \Psi(\kappa)\rangle=1$. The same shall hold for the natural orbitals and therefore also for all $|\boldsymbol{i}(\kappa)\rangle$. The expansion (6) is self-consistent in the sense that the respective expansion coefficients $c_{\boldsymbol{i}}(\kappa)$ fulfill self-consistency conditions, ensuring that the respective one-particle reduced density operator (5) is diagonal with respect to its own natural orbitals and that the natural occupation numbers are ordered non-increasingly.

As discussed in Appendix A, the self-consistent expansion (6) implies several convenient structural simplifications. First of all, a compact expression follows for the distance $D(\vec{\lambda}(\kappa))$ for all $\kappa$. To explain this, we employ second quantization using the natural orbitals of $|\Psi(\kappa)\rangle$ as reference basis. We can then express the natural occupation numbers as particle number expectation values, $\lambda_{j}(\kappa)=\left\langle\Psi(\kappa)\left|f^{\dagger}\left(\varphi_{j}(\kappa)\right) f\left(\varphi_{j}(\kappa)\right)\right| \Psi(\kappa)\right\rangle$, where $f^{\dagger}(\chi)$ and $f(\chi)$ create and annihilate a fermion in the state $|\chi\rangle$. Hence, by introducing for any GPC (1) the respective operator,

$$
\hat{D}_{\Psi(\kappa)} \equiv D\left(\left(\hat{n}_{j}(\kappa)\right)_{j=1}^{d}\right)
$$

$\hat{n}_{j}(\kappa) \equiv f^{\dagger}\left(\varphi_{j}(\kappa)\right) f\left(\varphi_{j}(\kappa)\right)$, we obtain (see also Ref. [9])

$$
D(\vec{\lambda}(\kappa))=\left\langle\Psi(\kappa)\left|\hat{D}_{\Psi(\kappa)}\right| \Psi(\kappa)\right\rangle .
$$

Moreover, one observes the elementary identity $\left\langle\boldsymbol{j}(\kappa)\left|\hat{D}_{\Psi(\kappa)}\right| \boldsymbol{i}(\kappa)\right\rangle=\delta_{\boldsymbol{j}, \boldsymbol{i}} D\left(\vec{e}_{\boldsymbol{i}}\right)$, where $\vec{e}_{\boldsymbol{i}}$ denotes a vector with entries $\left(\vec{e}_{\boldsymbol{i}}\right)_{k}=0,1$ depending on whether $k$ is contained in $\boldsymbol{i}$ (1) or not (0). This identity then immediately leads to the compact expression

$$
D(\vec{\lambda}(\kappa))=\sum_{\boldsymbol{i}}\left|c_{\boldsymbol{i}}(\kappa)\right|^{2} D\left(\vec{e}_{\boldsymbol{i}}\right) .
$$

It is remarkable that the right-hand side depends on $\kappa$ only via the coefficient functions $c_{\boldsymbol{i}}(\kappa)$. Moreover, 10 holds not only for GPCs but for any function $D$ linear in the natural occupation numbers $\lambda_{1}, \ldots, \lambda_{d}$. In the following, we consider only such GPCs which contain the
Hartree-Fock point $\vec{\lambda}_{H F} \equiv(1, \ldots, 1,0, \ldots, 0)$, i.e. constraints that are saturated for zero interaction, $\kappa \rightarrow 0$. By resorting to a perturbation theoretical expansion of the $c_{\boldsymbol{i}}(\kappa)$ and the natural orbitals $|j(\kappa)\rangle \equiv\left|\varphi_{j}(\kappa)\right\rangle$ one eventually finds (see derivation in Appendix A

$$
\begin{aligned}
D(\vec{\lambda}(\kappa))= & \kappa^{2} \sum_{\boldsymbol{i} \in \mathcal{I}_{2}}\left|\left\langle\boldsymbol{i}\left(0^{+}\right)\left|\left(\hat{H}^{(0)}-E^{(0)}\right)^{-1} \hat{V}\right| \boldsymbol{i}_{0}\right\rangle\right|^{2} D\left(\vec{e}_{\boldsymbol{i}}\right) \\
& +\mathcal{O}\left(\kappa^{3}\right)
\end{aligned}
$$

Here, the sum restricts to $\mathcal{I}_{2}$, the set of configurations $\boldsymbol{i}$ differing by exactly two orbital indices from $\boldsymbol{i}_{0} \equiv$ $(1,2, \ldots, N)$ and $E^{(0)}$ denotes the energy of the unperturbed ground state $|\Psi(0)\rangle=\left|\boldsymbol{i}_{0}\right\rangle$. Since $\rho_{1}(0)$ has a degenerate spectrum, the expression (11) involves the adapted unperturbed natural orbitals $\left|j\left(0^{+}\right)\right\rangle \equiv\left|\varphi_{j}\left(0^{+}\right)\right\rangle$ which do in general not coincide with the eigenstates $\left|\varphi_{j}\right\rangle$ of the one-particle Hamiltonian $\hat{H}^{(0)},\left|\varphi_{j}\left(0^{+}\right)\right\rangle \neq\left|\varphi_{j}\right\rangle$. The adapted natural orbitals, formally defined via the limit process $\kappa \rightarrow 0^{+}$, can be determined without much computational effort [39]. Hence, Eq. (11) defines a striking connection between the pair interaction of the physical system and the quantum information theoretical quantity $D(\vec{\lambda})$, quantifying the absolute influence of the fermionic exchange symmetry on the one-particle picture.

An additional comment is in order, emphasizing the significance of result (11). In general, after determining the adapted natural orbitals, one could implement a unitary basis set transformation from the one-particle eigenstates $\left|\varphi_{j}\right\rangle$ of $\hat{H}^{(0)}$ to those adapted states $\left|\varphi_{j}\left(0^{+}\right)\right\rangle$. This would change $\hat{V}$ to another pair interaction $\hat{V}^{\prime}$ and also $\hat{H}^{(0)}$ to another one-particle Hamiltonian with same energy spectrum . The respective expression (11) would then help to understand the mechanism behind quasipinning: The form of $\hat{V}^{\prime}$ is related in the simplest possible way to the leading order of the distance $D(\vec{\lambda}(\kappa))$ of $\vec{\lambda}(\kappa)$ to the polytope boundary.

\section{NUMERICALLY EXACT TREATMENT THROUGH DMRG}

To apply DMRG in the context of continuously confined fermions we use its quantum chemical version (QCDMRG) 40] adapted to spinless fermions and express Hamiltonian (2) in second quantization. As truncated reference basis we choose the first $d$ oscillator states $\left|\varphi_{j}\right\rangle$ of the external harmonic trap (now playing the role of the "lattice sites" in standard DMRG). The Hamiltonian then takes the form

$$
\hat{H}=\sum_{i, j=0}^{d-1} h_{i ; j} c_{i}^{\dagger} c_{j}+\frac{1}{2} K \sum_{i_{1}, i_{2}, j_{1}, j_{2}=0}^{d-1} V_{i_{1} i_{2} ; j_{1}, j_{2}} c_{i_{1}}^{\dagger} c_{i_{2}}^{\dagger} c_{j_{2}} c_{j_{1}}
$$

where (recall $m \equiv \omega \equiv \hbar \equiv 1$ )

$$
h_{i ; j} \equiv\left\langle\varphi_{i}\left|\hat{p}_{1}^{2} / 2+\hat{x}_{1}^{2} / 2\right| \varphi_{j}\right\rangle=\left(j+\frac{1}{2}\right) \delta_{i j}
$$


and

$$
V_{i_{1} i_{2} ; j_{1}, j_{2}} \equiv\left\langle\varphi_{i_{1}}\left|\otimes\left\langle\varphi_{i_{2}}|| \hat{x}_{1}-\left.\hat{x}_{2}\right|^{s} \mid \varphi_{j_{1}}\right\rangle \otimes\right| \varphi_{j_{2}}\right\rangle
$$

In a tedious derivation — being part of the long-term establishment of a DMRG scheme for systems of con- tinuously confined fermions 41] - one can determine an analytical expression for the two-particle matrix elements

$$
V_{i_{1} i_{2} ; j_{1}, j_{2}}=2^{s / 2} \frac{(-1)^{i_{2}+j_{2}}}{\sqrt{i_{1} ! i_{2} ! j_{1} ! j_{2} !}} \sum_{m_{1}=0}^{\min \left(i_{1}, j_{1}\right)} \sum_{m_{2}=0}^{\min \left(i_{2}, j_{2}\right)}\left(\begin{array}{c}
i_{1} \\
m_{1}
\end{array}\right)\left(\begin{array}{c}
j_{1} \\
m_{1}
\end{array}\right)\left(\begin{array}{c}
i_{2} \\
m_{2}
\end{array}\right)\left(\begin{array}{c}
j_{2} \\
m_{2}
\end{array}\right) m_{1} ! m_{2} ! \mathcal{J}_{i_{1}+i_{2}+j_{1}+j_{2}-2 m_{1}-2 m_{2}}
$$

assuming $i_{1}+i_{2}+j_{1}+j_{2}$ to be even since otherwise $V_{i_{1} i_{2} ; j_{1}, j_{2}}$ vanishes (recall the invariance of the pair interaction under spatial inversion). Here, $\mathcal{J}_{k} \equiv$ $\Gamma\left(\frac{s+1}{2}\right) \frac{2^{-k / 2}}{\sqrt{\pi}} \prod_{j=0}^{k / 2-1}(s-2 j)$ and $\Gamma$ denotes the Gamma function.

By choosing sufficiently large bases of up to $d=80$ orbitals, we ensure the convergence of both, the variational energy and the natural occupation numbers on at least ten digits. In particular, we use the dynamic block state selection (DBSS) procedure [42, 43] to reach a threshold accuracy of $10^{-13}$ in the energy. We also invoked the dynamically extended active space (DEAS) procedure 44] with a minimum number of block states set to $M=1024$ to guarantee fast and stable convergence during the initialization sweep of the DMRG. The residual error threshold for the respective Lánczos and Davidson diagonalization procedure is set to $10^{-13}$.

In the left panel of Fig. 11 we illustrate the convergence of our approach by comparing our variational energy to the exact one for the analytically solvable harmonic case [4], i.e., $s=2$, for the fixed coupling $\kappa=1$ and $N=2,3,4,5$ fermions. Convergence of the energy on more than ten digits is achieved in our approach by choosing $d$ sufficiently large. To illustrate the need for
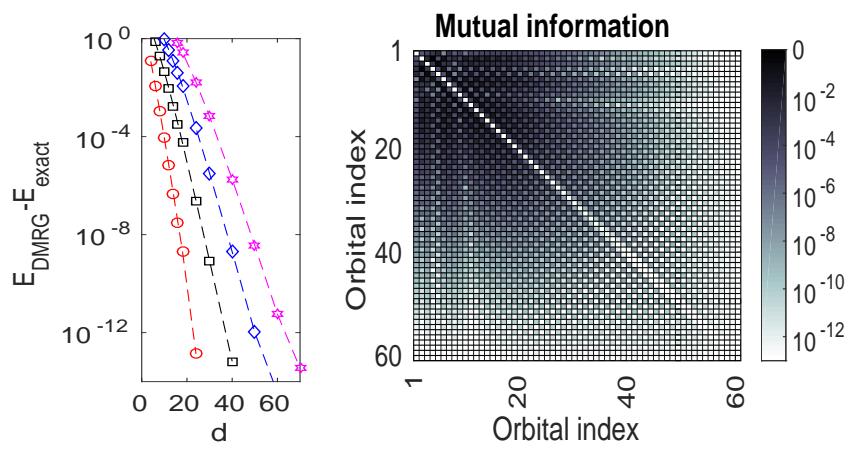

FIG. 1. Left: Absolute error of the DMRG ground state energy for $s=2$ (harmonic interaction), $\kappa=1$ as a function of the basis set size $d$. Cases $N=2,3,4,5$ are represented by symbols $\bigcirc, \square, \diamond, \star$, respectively and dashed lines emphasize the exponentially fast convergence in $d$. Right: Two-orbital mutual information $I_{i, j}$ for any two orbitals $i, j=1,2, \ldots, 60$ for same physical system and $N=5$. large basis sets from a different perspective, we present in the right panel of Fig. 1 for the same physical system the mutual information $I_{i, j}$ for any two orbitals $i, j=1,2, \ldots, 60$. Recall that $I_{i, j}$ quantifies the correlation between orbitals $i, j$ since it quantifies the extra information contained in the orbital reduced density operator $\rho_{i j}$ beyond the information already contained in both single orbital reduced density operators $\rho_{i}, \rho_{j}$, i.e. in $\rho_{i} \otimes \rho_{j}$ [4, 46, 49. While the orbitals around the Fermi level are apparently the most active ones, it can also be inferred from Fig. 1 that large basis sets, $d \gg N$, are required to cover also dynamical correlation up to high precision.

For a detailed presentation of the DMRG scheme which we developed to describe systems of continuously confined fermions and a comprehensive analysis of the entanglement structure of those systems we refer the reader to 41 .

\section{RESULTS}

For various numerically exact ground states calculated by DMRG we determine the corresponding one-particle reduced density matrices and diagonalize them numerically to obtain the natural occupation numbers $\lambda_{k}$. Since our high-precision approach involves large active spaces and since the GPCs are known so far only up to basis sets of size $d=12$ we resort to the concept of truncation 7. We perform the (quasi)pinning analysis in terms of a truncated vector $\vec{\lambda}^{\prime}$, obtained by discarding all NONs sufficiently close to 0 (and also 1 ). To be more specific, we quantify quasipinning by the minimal $l_{1}$-distance $D$ of $\vec{\lambda}$ to the polytope boundary. We then reduce $N$ to $N^{\prime}$ by ignoring eigenvalues close to 1 , and $d$ to $d^{\prime}$ by also ignoring those close to 0 . The minimal distance $D^{\prime}$ of $\vec{\lambda}^{\prime}$ to the boundary of the polytope $\mathcal{P}^{\prime}$ of $\left(N^{\prime}, d^{\prime}\right)$ coincides with $D$ in the full setting up to a truncation error $\varepsilon^{\prime}$,

$$
\left|D-D^{\prime}\right| \leq \varepsilon^{\prime} \equiv \sum_{j=1}^{N-N^{\prime}}\left(1-\lambda_{j}\right)+\sum_{k=0}^{d-d^{\prime}-N^{\prime}+N-1} \lambda_{d-k}
$$

Furthermore, since the polytope $\mathcal{P}$ is a subset of the Pauli simplex $1 \geq \lambda_{1} \geq \ldots \geq \lambda_{d} \geq 0$, quasipinning can be 
considered as non-trivial only if the distance of $\vec{\lambda}$ to the polytope boundary $\partial P$ is much smaller than the distance of $\vec{\lambda}$ to the boundary of the Pauli simplex. This "degree of non-triviality" is quantified by the $Q$-parameter [15].
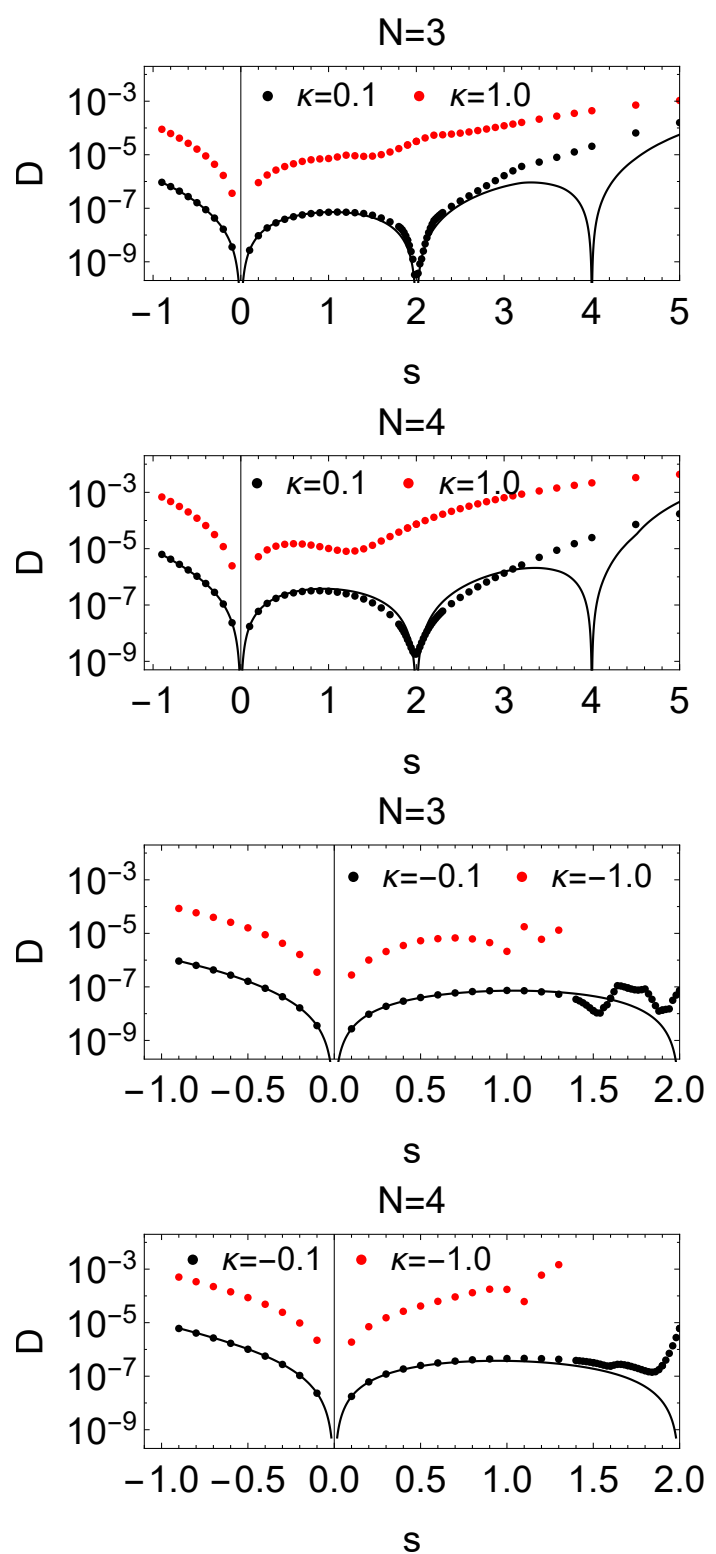

FIG. 2. For the ground state of the Hamiltonian 22 we present for different coupling strengths $\kappa$ the $s$-dependence of the minimal distance $D$ of the vector $\vec{\lambda}$ of NON to the polytope boundary. The quadratic leading order of $D$ following from the perturbation theory is shown as solid line.

In Fig. 2 we present the results for $D$ for a large grid of about $100 \mathrm{~s}$-values for the case of $N=3,4$ fermions for weak coupling $(\kappa= \pm 0.1)$ and medium coupling $(\kappa= \pm 1.0)$. For the case of negative couplings we restrict to $s<2$ since the system has no bound ground state for $s>2$. For all grid points we apply the "concept of truncation" [7] as explained above. The respective truncation errors for the particle numbers $N=3,4$ and the chosen couplings $\kappa$ turn out to be negligibly small, i.e. they are smaller than the width of the plot markers. Even for medium interaction strengths, those numerical results confirm that the GPCs have an absolute relevance for all $s$-values since the distance $D$ of the NONs $\vec{\lambda}$ to the polytope boundary is much smaller than the length scale of the polytope given by $\mathcal{O}(1)$. For the specific cases of a harmonic $(s=2)$ and quartic $(s=4)$ pair interaction the system exhibits an extremely strong quasipinning which is mainly due to the fact that the active space size shrinks for $s=2 n, n \in \mathbb{N}[7,11,13$. In that context, the reader should note that $s=0$ represents a non-interacting system implying $D(\vec{\lambda}(\kappa))=0$ for all couplings. A comparison of the results for small coupling $(|\kappa|=0.1)$ with those for medium coupling $(|\kappa|=1.0)$ shows that increasing the coupling leads to a weakening of the quasipinning which is due to the increase of the total correlation. Nonetheless, given that $D(\vec{\lambda}) \leq 10^{-3}$ for $|\kappa|=1.0$ for all $s$-values, the quasipinning for medium coupling is still quite strong.

Particularly remarkable is the fact that the unique nature of the extremely strong quasipinning in the neighbourhood of $s=2,4$ reduces a lot as one increases the coupling from very-small $(|\kappa| \ll 1)$ to small $(|\kappa|=0.1)$ and eventually medium coupling $(|\kappa|=1.0)$. In addition, it is also remarkable that for $\kappa= \pm 0.1$ the perturbation theoretical results for $D(\vec{\lambda})$ agree with the numerically exact DMRG results almost perfectly for $-1<s<2.5$ and $-1<s<1.3$ in case of $\kappa>0$ and $\kappa<0$, respectively. For $s>3$ the numerically exact results do not agree with the second-order perturbation theoretical results. This is due to the fact that for such extreme pair interactions, the coupling $\kappa=0.1$ is not yet small enough and thus higher orders $\left(\kappa^{3}\right)$ strongly affect the behaviour of $D(\lambda \overrightarrow{(\kappa)})$. Much better agreement between the numerical results and the perturbation theoretical results can be found for $s>3$, however, by considering smaller couplings as, e.g., $\kappa=0.01$ (not presented here).

While Fig. 2 confirms the relevance of the fermionic exchange symmetry on the one-particle picture, it is important to also understand to what extent this relevance needs to be assigned to Pauli's original principle. The potential significance of the GPCs beyond the already well-established relevance of Pauli's exclusion principle is quantified by the $Q$-parameter [15. Recall that a value $Q_{j}(\vec{\lambda})$ of the $Q$-parameter for the $j$-th GPC, $D_{j} \geq 0$, means that this GPC is $10^{Q_{j}(\vec{\lambda})}$ stronger saturated than what one could expect from the approximate saturation of some Pauli constraints. The results for the overall $Q$ parameter $Q=\max _{j}\left(Q_{j}\right)$ are presented in Fig. 3. First, we infer that the GPCs have a non-trivial relevance for all $s$-values and all considered $\kappa$-values, i.e. some GPCs are always saturated by a factor of about 10 stronger than Pauli's exclusion principle constraints would suggest. The singular behaviour of $D(\vec{\lambda})$ at $s=2,4$ is also present in the $Q$-parameter, at least for $s=2$. For 

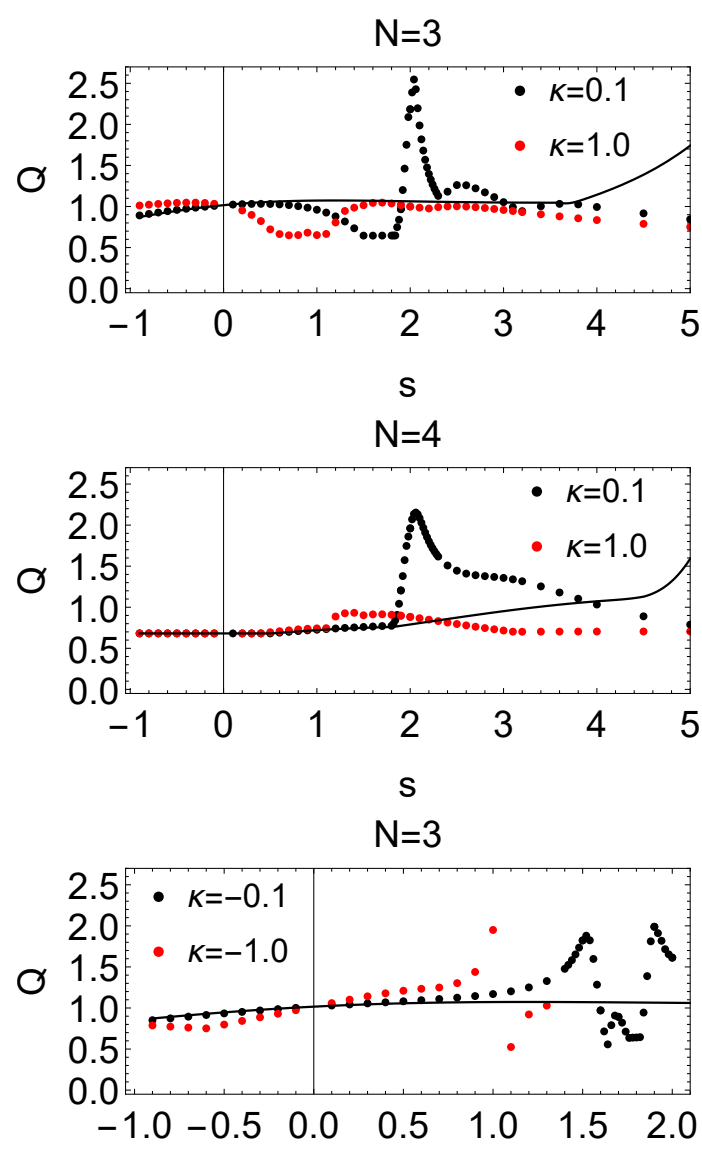

$\mathrm{S}$

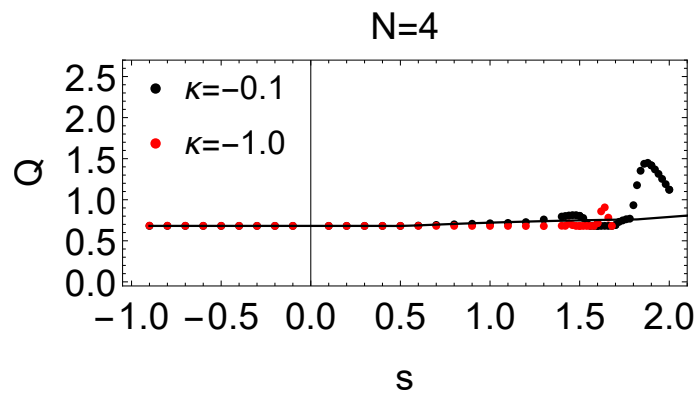

FIG. 3. For the ground state of the Hamiltonian (2) we present for different coupling strengths $\kappa$ the $s$-dependence of the $Q$-parameter. The leading order of $Q$ following from the perturbation theory is shown as solid line.

$s=4,6, \ldots$ the present DMRG results cannot resolve these singularities. Yet, we are convinced that the nontrivial character of the quasipinning is particularly pronounced at all positive even integer values of $s$, and in the respective vicinities. Furthermore, the perturbation theory (shown as solid line) shows that the leading (zeroth) order $Q^{(0)}$ of $Q(\vec{\lambda}(\kappa))$ is approximately constant for almost all $s$ in complete contrast to the harmonic case. Indeed, for $s=2$ and probably also for further eveninteger $s$-values above $s=2$ the $Q$-parameter diverges as the coupling $\kappa$ tends to zero (and thus does not allow for a perturbation theoretical expansion of $Q(\vec{\lambda}(\kappa)))$ around $\kappa=0$, i.e., $Q(\vec{\lambda}(\kappa))$ is not analytical at $\kappa=0$.

\section{SUMMARY AND CONCLUSION}

Conclusive results for a harmonic model system 7,11 13 provided first evidence that the generalized Pauli constraints (GPC) would have a tremendous physical relevance since they were found to be saturated up to a very small correction of the order eight in the coupling, $D \propto \kappa^{8}$. In the present work, we explore whether those seminal findings - the presence of such extremely strong quasipinning in ground states up to medium interaction strength - also hold for various other few-fermion systems. Answering this question is, however, highly challenging since the exact description of interacting fermions in the continuum can be considered as one of the hardest problems in physics. Even worse, as recent studies of atoms and molecules based on rather small active spaces have revealed, quasipinning found in those systems can be artificial in the sense that it is a mere consequence of the orbital and spin symmetries [13, 21, 25, 27. In our work, we largely avoided those bottlenecks by considering one-dimensional systems of spinless fermions interacting by a general pair potential of the form $\kappa\left|\hat{x}_{i}-\hat{x}_{j}\right|^{s}$ confined by a harmonic external potential. We resorted to the DMRG approach in the context of continuously confined fermions to determine the numerically exact ground states of those systems up to medium coupling $\kappa$ for the exemplary cases of $N=3,4$ fermions and for about 100 different pair interactions $-1<s \leq s_{\max }$, where $s_{\max }=5$ for attractive coupling $(\kappa>0)$ and $s_{\max }=2$ for repulsive coupling $(\kappa<0)$. By choosing sufficiently large basis sets of up to 80 orbitals we ensured the convergence on more than ten digits of both, the variational energy and the natural occupation numbers $\vec{\lambda} \equiv\left(\lambda_{i}\right)$.

Our numerically exact analysis confirms the original expectation: The GPCs are indeed universally relevant in the sense that they are approximately saturated regardless of the type of pair interaction. Even for medium interaction strength and almost the whole regime $-1<$ $s<5$ of considered pair interactions, we found a quasisaturation $D(\vec{\lambda}) \leq 10^{-3}$ of the GPCs. This provides further evidence that such quasipinning has its origin in the conflict between energy minimization and fermionic exchange symmetry which is present in all systems of continuously confined fermions. To distinguish between genuine and trivial quasipinning we used the $Q$-parameter [15]. The comprehensive analysis eventually confirms that the quasipinning by the GPCs is not primarily a result of the approximate saturation of Pauli's exclusion principle constraints $0 \leq \lambda_{j} \leq 1$.

To shed more light on the weak coupling regime, $|\kappa| \ll 1$, we developed a self-consistent perturbation theory which is based on an expansion of the $N$-fermion quantum states in Slater determinants built from its own natural orbitals. The respective analytical results 
agree quite well for most of the $s$-regime for coupling $\kappa= \pm 0.1$ with the numerical results. Furthermore, the analytical results also elucidate the singular behaviour found for the specific values $s=2,4, \ldots$, resulting in an even stronger, rather extreme quasipinning compared to generic $s$-values for weak couplings. In particular, the perturbation theory provides a conceptually important insight into quasipinning with consequences for the related terminology: Quasipinning in generic systems is quite strong despite the fact that the leading order correction is only quadratic, $D(\vec{\lambda}(\kappa))=c_{2} \kappa^{2}$. It is thus the respective prefactor $\left(c_{2}\right)$ rather than the exponent of the leading order of $D(\vec{\lambda}(\kappa))$ which defines the strength of quasipinning. Only for the specific case $s=2$ and probably some further even-integer $s$-values, the quadratic and further higher orders in the expansion of $D(\vec{\lambda}(\kappa))$ vanish rigorously.

All these results, presented in our work for $N=3,4$ fermions, hold qualitatively also for larger particle numbers.

Due to the remarkable implication of (quasi)pinning for the structure of the many-fermion wave functions [10], our findings emphasize again the potential significance that GPCs may have in few-body quantum systems, particularly for Multi-Configurational Self-Consistent Field (MCSCF) ansatzes and in Reduced Density Matrix Functional Theory (RDMFT). Such applications of the GPCs would require, however, that the recent development [46, 24, 50, 58, in quantum information sciences and mathematical physics yields more efficient algorithms for the calculations of the GPCs for larger active spaces.

\section{ACKNOWLEDGMENTS}

We acknowledge financial support from the Oxford Martin Programme on Bio-Inspired Quantum Technologies and the UK Engineering and Physical Sciences Research Council (Grant EP/P007155/1) (C.S.), the Hungarian National Research, Development and Innovation Office (NKFIH) through Grant No. K120569 and the Hungarian Quantum Technology National Excellence Program (Project No. 2017-1.2.1-NKP-201700001) (O.L.)

\section{Appendix A: Details of the self-consistent perturbation theory}

In this appendix we present all technical details of the self-consistent perturbation theory proposed in Sec. II A.

Let us consider an $N$-particle Hamiltonian of the general form

$$
\hat{H}(\kappa)=\hat{H}^{(0)}+\kappa \hat{V}
$$

acting on the $N$-fermion Hilbert space $\wedge^{N}\left[\mathcal{H}_{1}^{(d)}\right]$, where $\mathcal{H}_{1}^{(d)}$ denotes the underlying one-particle Hilbert space of dimension $d$. Here, $\hat{H}^{(0)}$ is a general one-particle Hamiltonian including the kinetic energy and the external potential and $\hat{V}$ is a pair interaction. We assume that the ground state $|\Psi(\kappa)\rangle$ of the Hamiltonian A1 depends analytically on $\kappa$, at least in a neighbourhood of $\kappa=0$, and thus allows us to study it by perturbation theoretical means around $\kappa=0$.

We expand $|\Psi(\kappa)\rangle$ self-consistently according to (6). The respective natural orbitals $\left|\varphi_{j}(\kappa)\right\rangle \equiv|j(\kappa)\rangle$ follow from the one-particle reduced density operator (5) obtained after tracing out $N-1$ fermions. This selfconsistent expansion has a couple of convenient properties. To discuss them we expand the coefficient functions $c_{i}(\kappa)$

$$
c_{\boldsymbol{i}}(\kappa)=c_{\boldsymbol{i}}^{(0)}+\kappa c_{\boldsymbol{i}}^{(1)}+\mathcal{O}\left(\kappa^{2}\right)
$$

and the natural orbitals $|j(\kappa)\rangle$,

$$
|j(\kappa)\rangle=\left|j^{(0)}\right\rangle+\kappa\left|j^{(1)}\right\rangle+\mathcal{O}\left(\kappa^{2}\right) .
$$

Here, the natural orbitals (and thus the Slater determinants $|\boldsymbol{j}(\kappa)\rangle)$ shall be normalized to unity for all $\kappa$, $\langle j(\kappa) \mid j(\kappa)\rangle=1$. It is important to notice that the states $\left|j^{(0)}\right\rangle \equiv \lim _{\kappa \rightarrow 0^{+}}|j(\kappa)\rangle$ do in general not coincide with the one-particle eigenstates of the unperturbed Hamiltonian $\hat{H}^{(0)}$. Nonetheless, one has

$$
\begin{gathered}
\operatorname{span}\left(\{|j\rangle\}_{1 \leq j \leq N}\right)=\operatorname{span}\left(\left\{\left|j^{(0)}\right\rangle\right\}_{1 \leq j \leq N}\right) \\
\operatorname{span}\left(\{|j\rangle\}_{N+1 \leq j \leq d}\right)=\operatorname{span}\left(\left\{\left|j^{(0)}\right\rangle\right\}_{N+1 \leq j \leq d}\right)
\end{gathered}
$$

Concerning the total quantum state (6), the expansion in $\kappa$ reads

$$
|\Psi(\kappa)\rangle=\left|\Psi^{(0)}\right\rangle+\kappa\left|\Psi^{(1)}\right\rangle+\mathcal{O}\left(\kappa^{2}\right)
$$

with the normalization condition $\langle\Psi(\kappa) \mid \Psi(\kappa)\rangle=1$. Since the ground state is assumed to be unique, we have

$$
c_{\boldsymbol{i}}^{(0)}= \begin{cases}1, & \text { if } \boldsymbol{i}=\boldsymbol{i}_{0} \\ 0, & \text { otherwise }\end{cases}
$$

where $\boldsymbol{i}_{0} \equiv(1,2, \ldots, N)$. A first useful property of the self-consistent expansion (6) is that configurations $\boldsymbol{i}$ differing by exactly one orbital index from the referenceconfiguration $\boldsymbol{i}_{0} \equiv(1,2, \ldots, N)$ contribute to $|\Psi(\kappa)\rangle$ with significantly reduced weight as stated in the following Lemma.

Lemma 1. Let $|\Psi(\kappa)\rangle$ be an $N$-fermion quantum state, analytical in $\kappa$, and normalized to unity, $\langle\Psi(\kappa) \mid \Psi(\kappa)\rangle=$ 1. We denote the overall first contribution in the series of the analytical coefficient functions $c_{\boldsymbol{j}}(\kappa)$ in the self-consistent perturbation expansion (6) by $r$. Then, $c_{\boldsymbol{i}_{0}}(\kappa)=1+\mathcal{O}\left(\kappa^{2 r}\right)$ and all configurations $\boldsymbol{i}$ differing from the reference configuration $\boldsymbol{i}_{0} \equiv(1,2, \ldots, N)$ by exactly one orbital index, $\left|\boldsymbol{i} \cap \boldsymbol{i}_{0}\right|=N-1$, contribute only with weight of the order $\mathcal{O}\left(\kappa^{2 r}\right)$. 
Proof. Consider a configuration $\boldsymbol{i}$ differing from the reference configuration $\boldsymbol{i}_{0}$ by the orbital index $\alpha>N$ which replaces the index $l \leq N$ in $\boldsymbol{i}_{0}$, i.e. $\boldsymbol{i}=\left(\boldsymbol{i}_{0} \cup\{\alpha\}\right) \backslash\{l\}$ Due to the self-consistent character of the expansion (6), the one-particle reduced density operator $\rho_{1}(\kappa)$ is diagonal in the basis of its own natural orbitals. In particular,

$$
\begin{aligned}
0 & \stackrel{!}{=}\left\langle\alpha(\kappa)\left|\rho_{1}(\kappa)\right| l(\kappa)\right\rangle \\
& =\sum_{\boldsymbol{j} \ni l} c_{\boldsymbol{j}}^{*}(\kappa) c_{(\boldsymbol{j} \cup\{\alpha\}) \backslash\{l\}}(\kappa)(-1)^{\#\{i \in \boldsymbol{j} \mid k<i<\alpha\}} \\
& =c_{\boldsymbol{i}_{0}}^{*}(\kappa) c_{\boldsymbol{i}}(\kappa)(-1)^{N-l+1}+\mathcal{O}\left(\kappa^{2 r}\right) .
\end{aligned}
$$

In the last line, we have used for $\boldsymbol{i} \neq \boldsymbol{i}_{0}$ that $c_{\boldsymbol{i}}(\kappa)=$ $\mathcal{O}\left(\kappa^{r}\right)$, i.e., $r$ is the overall leading order in $\kappa$ of the series A2 . Finally, by using $c_{\boldsymbol{i}_{0}}(\kappa)=1+\mathcal{O}\left(\kappa^{2 r}\right)$ following from the normalization $1=\langle\Psi(\kappa) \mid \Psi(\kappa)\rangle=\left|c_{\boldsymbol{i}_{0}}(\kappa)\right|^{2}+\mathcal{O}\left(\kappa^{2 r}\right)$ we then obtain from A7 $c_{\boldsymbol{i}}(\kappa)=\mathcal{O}\left(\kappa^{2 r}\right)$.

It is worth noticing that for generic Hamiltonians of the form (4) the leading order corrections are of linear order, $r=1$. For special cases, as, e.g., the Harmonium model defined by Hamiltonian (2) with $s=2$, however, one can find $r>1$.

To determine the first order contributions $c_{i}^{(1)}$ to the quantum state (6) we study the respective timeindependent Schrödinger equation

$$
E(\kappa)|\Psi(\kappa)\rangle=\left(\hat{H}^{(0)}+\kappa \hat{V}\right)|\Psi(\kappa)\rangle
$$

In zeroth order, by expanding the energy according to

$$
E(\kappa) \equiv E^{(0)}+\kappa E^{(1)}+\mathcal{O}\left(\kappa^{2}\right)
$$

we have

$$
E^{(0)}\left|\Psi^{(0)}\right\rangle=\hat{H}^{(0)}\left|\Psi^{(0)}\right\rangle
$$

This apparently yields (recall also A4)

$$
\left|\Psi^{(0)}\right\rangle=\left|\boldsymbol{i}_{0}\right\rangle=\left|\boldsymbol{i}_{0}\left(0^{+}\right)\right\rangle, \quad E^{(0)}=E_{\boldsymbol{i}_{0}},
$$

where for any configuration $\boldsymbol{i} \equiv\left(i_{1}, \ldots, i_{N}\right), E_{\boldsymbol{i}}$ denotes the unperturbed energy of the respective Slater determinant $|\boldsymbol{i}\rangle . E_{\boldsymbol{i}}$ is nothing else than just the sum of the single-particle energies of the orbitals $\left|i_{1}\right\rangle, \ldots\left|i_{N}\right\rangle$. Moreover, we have indeed $\left|\boldsymbol{i}_{0}\right\rangle=\left|\boldsymbol{i}_{0}\left(0^{+}\right)\right\rangle$(up to a phase which we can set to zero) which follows from (A4).

In linear order, A8 leads to

$$
E^{(1)}\left|\Psi^{(0)}\right\rangle+E^{(0)}\left|\Psi^{(1)}\right\rangle=\hat{H}^{(0)}\left|\Psi^{(1)}\right\rangle+\hat{V}\left|\Psi^{(0)}\right\rangle
$$

By projecting A12 onto $\left|\Psi^{(0)}\right\rangle=\left|\boldsymbol{i}_{0}\left(0^{+}\right)\right\rangle=\left|\boldsymbol{i}_{0}\right\rangle$ and using A10 we find

$$
E^{(1)}=\left\langle\boldsymbol{i}_{0}|\hat{V}| \boldsymbol{i}_{0}\right\rangle
$$

On the orthogonal complement of $\left|\boldsymbol{i}_{0}\left(0^{+}\right)\right\rangle$we can invert the operator $E^{(0)}-\hat{H}^{(0)}$. Thus we obtain from A12 restricted to $\operatorname{span}\left(\left\{\left|\boldsymbol{i}\left(0^{+}\right)\right\rangle\right\}_{\boldsymbol{i} \neq \boldsymbol{i}_{0}}\right)$

$$
\left|\Psi^{(1)}\right\rangle=\left(E^{(0)}-\hat{H}^{(0)}\right)^{-1} \hat{V}\left|\Psi^{(0)}\right\rangle .
$$

Moreover, by comparing A2 and A3 with A5 and using A6 and Lemma 1 we find

$$
\begin{aligned}
\left|\Psi^{(1)}\right\rangle= & \sum_{\boldsymbol{i}}\left[c_{\boldsymbol{i}}^{(1)}\left|\boldsymbol{i}\left(0^{+}\right)\right\rangle+c_{\boldsymbol{i}}^{(0)}|\boldsymbol{i}(\kappa)\rangle^{(1)}\right] \\
& \sum_{\boldsymbol{i} \in \mathcal{I}_{\geq 2}} c_{\boldsymbol{i}}^{(1)}\left|\boldsymbol{i}\left(0^{+}\right)\right\rangle+\left|\boldsymbol{i}_{0}(\kappa)\right\rangle^{(1)} .
\end{aligned}
$$

Here, $|\boldsymbol{i}(\kappa)\rangle^{(1)}$ denotes the linear order of $|\boldsymbol{i}(\kappa)\rangle$ in $\kappa$ and $\mathcal{I}_{\geq 2}$ denotes the set of all configurations $\boldsymbol{i}=\left(i_{1}, \ldots, i_{N}\right)$ differing in at least two indices from $\boldsymbol{i}_{0} \equiv(1, \ldots, N)$.

Since $\left\langle\boldsymbol{i}\left(0^{+}\right) \mid \boldsymbol{i}_{0}(\kappa)\right\rangle^{(1)}=0$ for all $\boldsymbol{i}$ differing from $\boldsymbol{i}_{0}$ by more than one orbital index, A15 yields

$$
\left\langle\boldsymbol{i}\left(0^{+}\right) \mid \Psi^{(1)}\right\rangle=c_{\boldsymbol{i}}^{(1)}, \quad \forall \boldsymbol{i} \in \mathcal{I}_{\geq 2}
$$

Using this in combination with A14 leads to

$$
c_{\boldsymbol{i}}^{(1)}=\left\langle\boldsymbol{i}\left(0^{+}\right)\left|\left(E^{(0)}-\hat{H}^{(0)}\right)^{-1} \hat{V}\right| \boldsymbol{i}_{0}\right\rangle
$$

for all $\boldsymbol{i} \in \mathcal{I}_{\geq 2}$. Finally, we observe that according to A17), $c_{\boldsymbol{i}}^{(1)}$ also vanishes in case $\boldsymbol{i}$ differs from $\boldsymbol{i}_{0}$ by more than two indices since $\hat{V}$ is a two-particle operator. Hence, 11 follows from (10) by using (A17).
[1] W. Pauli, "Über den Zusammenhang des Abschlusses der Elektronengruppen im Atom mit der Komplexstruktur der Spektren," Z. Phys. 31, 765-783 (1925).

[2] R.E. Borland and K. Dennis, "The conditions on the onematrix for three-body fermion wavefunctions with onerank equal to six," J. Phys. B 5, 7 (1972).

[3] M. B. Ruskai, "Connecting N-representability to Weyl's problem: the one-particle density matrix for $\mathrm{N}=3$ and $\mathrm{R}=6, "$ J. Phys. A 40, F961 (2007)

[4] A. Klyachko, "Quantum marginal problem and N- representability," J. Phys. Conf. Ser. 36, 72 (2006).

[5] M. Altunbulak and A. Klyachko, "The Pauli principle revisited," Commun. Math. Phys. 282, 287-322 (2008).

[6] M. Altunbulak, The Pauli principle, representation theory, and geometry of flag varieties, Ph.D. thesis, Bilkent University (2008).

[7] F. Tennie, D. Ebler, V. Vedral, and C. Schilling, "Pinning of fermionic occupation numbers: General concepts and one spatial dimension," Phys. Rev. A 93, 042126 (2016) 
[8] A. Klyachko, "The Pauli exclusion principle and beyond," arXiv:0904.2009 (2009)

[9] C. Schilling, "Quasipinning and its relevance for $N$ fermion quantum states," Phys. Rev. A 91, 022105 (2015)

[10] C. Schilling, C. L. Benavides-Riveros, and P. Vrana, "Reconstructing quantum states from single-party information," Phys. Rev. A 96, 052312 (2017)

[11] C. Schilling, D. Gross, and M. Christandl, "Pinning of fermionic occupation numbers," Phys. Rev. Lett. 110, 040404 (2013)

[12] D. Ebler, Pinning analysis for 4-harmonium (Semester thesis, ETH Zurich, 2013).

[13] C. Schilling, Quantum marginal problem and its physical relevance, Ph.D. thesis, ETH-Zürich (2014).

[14] F. Tennie, V. Vedral, and C. Schilling, "Pinning of fermionic occupation numbers: Higher spatial dimensions and spin," Phys. Rev. A 94, 012120 (2016).

[15] F. Tennie, V. Vedral, and C. Schilling, "Influence of the fermionic exchange symmetry beyond Pauli's exclusion principle," Phys. Rev. A 95, 022336 (2017).

[16] F. Tennie, Influence of the exchange symmetry beyond the exclusion principle, Ph.D. thesis, University of Oxford (2017).

[17] C. L. Benavides-Riveros, J. M. Gracia-Bondía, and M. Springborg, "Quasipinning and entanglement in the lithium isoelectronic series," Phys. Rev. A 88, 022508 (2013)

[18] A. Klyachko, "The Pauli principle and magnetism," arXiv:1311.5999 (2013)

[19] R. Chakraborty and D.A. Mazziotti, "Generalized Pauli conditions on the spectra of one-electron reduced density matrices of atoms and molecules," Phys. Rev. A 89, 042505 (2014)

[20] R. Chakraborty and D.A. Mazziotti, "Sufficient condition for the openness of a many-electron quantum system from the violation of a generalized Pauli exclusion principle," Phys. Rev. A 91, 010101 (2015).

[21] C. L. Benavides-Riveros and M. Springborg, "Quasipinning and selection rules for excitations in atoms and molecules," Phys. Rev. A 92, 012512 (2015).

[22] I. Theophilou, N.N. Lathiotakis, M. Marques, and N. Helbig, "Generalized Pauli constraints in reduced density matrix functional theory," J. Chem. Phys. 142, 154108 (2015)

[23] R. Chakraborty and D. A. Mazziotti, "Structure of the one-electron reduced density matrix from the generalized Pauli exclusion principle," Int. J. Quant. Chem. 115, 1305-1310 (2015)

[24] A. Lopes, Pure univariate quantum marginals and electronic transport properties of geometrically frustrated systems, Ph.D. thesis, University of Freiburg (2015).

[25] C. Schilling, "Hubbard model: Pinning of occupation numbers and role of symmetries," Phys. Rev. B 92, 155149 (2015)

[26] J. Wang and P.J. Knowles, "Nonuniqueness of algebraic first-order density-matrix functionals," Phys. Rev. A 92, 012520 (2015)

[27] C. L. Benavides-Riveros, Disentangling the marginal problem in quantum chemistry, Ph.D. thesis, Universidad de Zaragoza (2015).

[28] R. Chakraborty and D. A. Mazziotti, "Role of the generalized Pauli constraints in the quantum chemistry of excited states," Int. J. Quant. Chem. 116 (2016).
[29] D.A. Mazziotti, "Pure- $n$-representability conditions of two-fermion reduced density matrices," Phys. Rev. A 94, 032516 (2016).

[30] C. L. Benavides-Riveros, N. N. Lathiotakis, and M. A. L. Marques, "Towards a formal definition of static and dynamic electronic correlations," Phys. Chem. Chem. Phys. 19, 12655 (2017)

[31] R. Chakraborty and D.A. Mazziotti, "Noise-assisted energy transfer from the dilation of the set of one-electron reduced density matrices," J. Chem. Phys. 146, 184101 (2017)

[32] C. Schilling, M. Altunbulak, S. Knecht, A. Lopes, J. D. Whitfield, M. Christandl, D. Gross, and M. Reiher, "Generalized Pauli constraints in small atoms," arXiv:1710.03074 (2017)

[33] T. Maciazek and V. Tsanov, "Quantum marginals from pure doubly excited states," J. Phys. A 50, 465304 (2017)

[34] I. Bloch, J. Dalibard, and W. Zwerger, "Many-body physics with ultracold gases," Rev. Mod. Phys. 80, 885964 (2008)

[35] C. Chin, R. Grimm, P. Julienne, and E. Tiesinga, "Feshbach resonances in ultracold gases," Rev. Mod. Phys. 82, 1225-1286 (2010).

[36] M. Weidemüller and C. Zimmermann, Interactions in ultracold gases: from atoms to molecules (John Wiley \& Sons, 2011).

[37] S.R. White, "Density matrix formulation for quantum renormalization groups," Phys. Rev. Lett. 69, 2863-2866 (1992)

[38] P.O. Löwdin, "Quantum theory of many-particle systems. I. physical interpretations by means of density matrices, natural spin-orbitals, and convergence problems in the method of configurational interaction," Phys. Rev. 97, 1474 (1955)

[39] E.R. Davidson, Reduced Density Matrices in Quantum Chemistry (Academic Press, New York, 1976).

[40] S. White and R. Martin, "Ab initio quantum chemistry using the density matrix renormalization group," J. Chem. Phys. 110, 4127-4130 (1999)

[41] O. Legeza and C. Schilling, "QC-DMRG for harmonically confined fermions with arbitrary pair interactions," In preparation.

[42] Ö. Legeza, J. Röder, and B. A. Hess, "Controlling the accuracy of the density-matrix renormalization-group method: The dynamical block state selection approach," Phys. Rev. B 67, 125114 (2003).

[43] O. Legeza and J. Sólyom, "Quantum data compression, quantum information generation, and the densitymatrix renormalization-group method," Phys. Rev. B 70, 205118 (2004)

[44] O. Legeza and J. Sólyom, "Optimizing the density-matrix renormalization group method using quantum information entropy," Phys. Rev. B 68, 195116 (2003).

[45] Z.-L. Wang, A.M. Wang, Y. Yang, and X.C. Li, "Exact eigenfunctions of n-body system with quadratic pair potential," Comm. Theor. Phys. 58, 639 (2012).

[46] Ö. Legeza and J. Sólyom, "Two-site entropy and quantum phase transitions in low-dimensional models," Phys. Rev. Lett. 96, 116401 (2006).

[47] J. Rissler, R. Noack, and S.R. White, "Measuring orbital interaction using quantum information theory," Chem. Phys. 323, 519 - 531 (2006) 
[48] G. Barcza, Ö. Legeza, K. H. Marti, and M. Reiher, "Quantum-information analysis of electronic states of different molecular structures," Phys. Rev. A 83, 012508 (2011)

[49] S. Szalay, M. Pfeffer, V. Murg, G. Barcza, F. Verstraete, R. Schneider, and Ö. Legeza, "Tensor product methods and entanglement optimization for ab initio quantum chemistry," Int. J. Quant. Chem. 115, 1342 (2015).

[50] A. Sawicki and Kus M., "Geometry of the local equivalence of states," J. Phys. A 44 (2011)

[51] A. Sawicki, M. Oszmaniec, and M. Kus, "Critical sets of the total variance of state detect all slocc entanglement classes," Phys. Rev. A 86, 040304(R) (2012).

[52] A. Sawicki and V. Tsanov, "A link between quantum entanglement, secant varieties and sphericity," J. Phys. A 46 (2013).

[53] T. Maciazek, M. Oszmaniec, and A. Sawicki, "How many invariant polynomials are needed to decide local unitary equivalence of qubit states?" J. Math. Phys. 54, 092201
(2013)

[54] A. Huckleberry, Kus M., and Sawicki A., "Bipartite entanglement, spherical actions, and geometry of local unitary orbits," J. Math. Phys. 54, 022202 (2013)

[55] A. Sawicki, M. Oszmaniec, and M. Kus, "Convexity of momentum map, morse index, and quantum entanglement," Rev. Math. Phys. 26, 1450004 (2014).

[56] T. Maciazek and A. Sawicki, "Critical points of the linear entropy for pure l-qubit states," J. Phys. A 48, 045305 (2015)

[57] A. Sawicki, T. Maciazek, M. Oszmaniec, K. Karnas, K. Kowalczyk-Murynka, and M. Kus, "Multipartite quantum correlations: symplectic and algebraic geometry approach," arXiv:1701.03536 (2017).

[58] T. Maciazek and A. Sawicki, "Asymptotic properties of entanglement polytopes for large number of qubits," arXiv:1706.05019 (2017) 